\title{
Competence and Preference-based Workplace Assignment
}

An Application of a Weighted, Non-pre-emptive and

Pre-emptive Goal Programming Model

\author{
Malte L. Peters and Stephan Zelewski \\ University of Duisburg-Essen, Institute of Production and Industrial Information \\ Management, Universitaetsstrasse 10, D-45141 Essen, Germany. \\ Email: \{malte.peters,stephan.zelewski\}@pim.uni-essen.de
}

\begin{abstract}
Employees should be assigned to workplaces according to their competences and preferences to ensure motivated employees carrying out tasks effectively and efficiently. This contribution presents a goal programming model for workplace assignment, which takes into account both employee competences and preferences and workplace competence requirements and attributes.
\end{abstract}

Key words: Goal programming, Workplace and employee assignment, Competences

\section{INTRODUCTION: PROBLEM IDENTIFICATION}

In practice, employee workplace assignment can lead to ineffective and inefficient job performance for several reasons. On the one hand, employees probably might not satisfy the competence requirements associated with the workplaces to which they have been assigned and, as a result, they cannot cope with workplace strains. Employees should therefore be assigned to workplaces according to their competences to ensure effective and efficient job performance. On the other hand, employees may have been assigned to their workplaces against their preferences regarding the competences and the general conditions of the workplace.

Non-fulfilment of competence requirements as well as assignment contrary to employee preference may easily lead to employee demotivation. Firstly, workplace assignment based upon individual competences enables 
the employees to select the appropriate activities to perform the tasks. As a result, they are able to complete their tasks more easily. Secondly, the consideration of competence preferences leads to higher motivation since employees are normally more motivated to complete tasks related to their interests and abilities. Moreover, this consideration affords employees the opportunity to rank their interests and abilities. An employee might prefer to speak Spanish rather than French, for example. Such an employee would prefer a workplace with a higher relative importance of the competence "Spanish language knowledge" in comparison to the competence "French language knowledge". Thirdly, the consideration of preferences regarding general workplace conditions also leads to higher motivation. A mother raising children on her own may prefer a workplace with a reduced number of work hours per day or reduced work days in a week.

\section{PRELIMINARIES}

\subsection{Terminological Preliminaries}

\subsubsection{Competences, Competence Hierarchies, Competence Profiles, and Competence Levels}

A competence is defined as the ability of an employee to use his or her knowledge to achieve a predefined goal, such as an effective and efficient execution of a task. An example of a competence is an employee's ability to use his or her language knowledge to negotiate with foreign business partners. Competences can be structured in a so-called competence hierarchy. In such a hierarchy, similar competences are grouped under a node. The competences "French language knowledge" and "Spanish language knowledge", for example, may be grouped under the node "Foreign language knowledge". Moreover, competence profiles are suitable for competence documentation. On the one hand, employee competence profiles are required to document the competences of each employee. An employee's competence profile consists of a certain set of competences and an assessment of how well an employee meets each competence. The competences require assessment because the level of proficiency in each competence (employee competence level) may vary from employee to employee. One employee might reach a higher level of proficiency in the competence "French language knowledge" than another, for example. How well an employee meets each competence is assessed using an ordinal scale with levels ranging from 1 (dilettante) to 5 (expert). Other scales may be more appropriate (e.g., scales with 9 levels), depending on the desired degree of detail. ERP Systems (e.g., SAP R/3) and 
project management systems (e.g., Primavera P3e) provide appropriate functions. On the other hand, workplace requirement profiles are needed to document the minimum or specific fixed competences required for each workplace. A requirement profile has to be created for each workplace using the same ordinal scale. Each workplace requirement profile encloses a minimum or specific fixed level of proficiency for each competence. Moreover, a workplace requirement profile contains an assessment of the importance of a competence to a workplace.

\subsubsection{Workplace Attributes, Attribute Hierarchies, and Attribute Profiles}

Workplace attributes describe the general conditions associated with a workplace: "hours of work per day", "number of work days in a week", "operational safety", "building security", and "office space" serve as examples of such attributes. Analogous to competences, workplace attributes can be structured in an attribute hierarchy. The workplace attributes "building security", and "office space" may be grouped under the node "location attributes". All workplace attributes with the accompanying attribute values describing a certain workplace can be combined into a workplace attribute profile. The preferences of each employee for specific attribute values can be combined into an employee attribute profile in the same way.

\subsection{Methodological Preliminaries}

\subsubsection{Analytical Hierarchy Process}

The goal programming model presented here requires several assessments such as competence importance to the specific workplaces. These assessments can be undertaken using the analytical hierarchy process (AHP; e.g., SAATY 2001). The standard AHP requires paired comparison judgments concerning the dominance of one element (e.g. to assess the importance of a competence to a workplace) over another for each of a set of elements using a 1-9 scale, in order to obtain an importance weight for each element. The paired comparison judgments are entered in a square matrix $A$. If an element $i$ is judged to be moderately important by comparison with another element $j$, for example, a 3 is entered as the value for the paired comparison judgment $a_{i j}$ in the matrix $A$ while the reciprocal value is entered for the paired comparison judgment $a_{j i}$. The importance weights are derived by computing a normalised eigenvector of the matrix $A$. Making pairwise comparison judgments in a model that has a large number of elements (e.g. competences, preferences) can be very time consuming. 
Accordingly, if a large number of elements is considered, it could be practical to create a hierarchy of the elements. While a hierarchy of the elements can be used to reduce the number of pairwise comparisons, it can also be helpful to have well-structured elements. Further refinements of the AHP are addressed in the specialised literature (e.g., SAATY 2001).

\subsubsection{Goal Programming}

Goal programming is a mathematical programming technique designed to handle multiple conflicting objectives. CHARNES and COOPER (1961, p. 215 ) introduced goal programming. The utilisation of its models has spread in many, diversified fields of interest, such as site selection (HOFFMAN, SCHNIEDERJANS 1994), and project selection (MUKHERJEE, BERA 1995). In goal programming, each objective is viewed as a goal. The technique enables a decision maker to consider one-sided goals and two-sided goals. If the objective is to reach or exceed a one-sided goal, it is called an upper one-sided goal; otherwise, if the objective is to reach or fall below a one-sided goal, it is called a lower one-sided goal. If the objective is to meet a goal, it is called a two-sided goal (HILLIER, LIEBERMAN 2001, p. 332).

The aim of the application of goal programming is to minimise the deviations of the goals considered. So-called deviational variables measure the amount by which the values delivered by the solution of the goal programming model deviate from the respective goal. If an upper (a lower) onesided goal is considered, the objective function will contain a non-negative underachievement (overachievement) variable which measures the amount of failing to reach or exceed (or fall below) the desired goal. If a two-sided goal is considered, the objective function will contain both an underachievement and an overachievement variable. Further, the basic goal programming model can be enhanced by considering differences in the relative importance of goals. This enhanced approach is named weighted goal programming and assigns importance to the underachievement or overachievement variables according to their relative importance.

Moreover, two types of goal programming models are differentiated: the pre-emptive case and the non-pre-emptive case (e.g., HILLIER, LIEBERMAN 2001, pp. 333-339). In pre-emptive goal programming models different objectives are prioritised as first-priority-goals, second-priority-goals and so on. In an initial step a first goal programming model is solved which only incorporates the first-priority-goals. If the execution of the initial step leads to more than one optimal solution of the first model, a second model incorporating the second-priority-goals is solved keeping the optimal achievement-level of first-priority-goals constant. As long as the execution of a step leads to more than one optimal solution of the respective model and as long as there are goals of lower priority defined, a further model is applied in an 
additional step. Lower-priority-goals are not considered unless the higherpriority-goals are optimally satisfied and this optimal solution is many-valued. By contrast, in non-pre-emptive goal programming models, different objectives are considered simultaneously as goals in an aggregated objective function. In the non-pre-emptive case, different importance can also be assigned both to the different objectives and to individual goals.

\section{MODEL FORMULATION}

\subsection{Input Data Preparation}

The goal programming model presented here makes it possible to consider three different objectives:

- assignment of employees to workplaces according to the fit of their actual and the required levels of competence, respectively;

- assignment of the employees to workplaces according to the fit of their preferences regarding the competences and the relative importance of the competences to the workplaces;

- assignment of the employees to workplaces according to the fit of their preferences regarding the general conditions of the workplace and the actual values of workplace attributes.

The first step is to assess the relative importance of the objectives employing the AHP. In the pre-emptive case presented below, the first objective is considered as single first-priority-goal, while the second and the third objectives are considered as second-priority-goals. The pre-emptive case requires the assessment of the relative importance $v_{2}$ and $v_{3}$ of the second and the third objective. In the non-pre-emptive case all three objectives are considered simultaneously as first-priority-goals. Thus it requires the assessment of the relative importance $v_{1}, v_{2}$, and $v_{3}$ of all three objectives. If an objective is not to be considered, it has to be assigned a value of 0 to the respective importance $v_{1}, v_{2}$, or $v_{3}$. Only the pre-emptive case enforces the consideration of the first objective. If the first objective is not to be considered, the non-pre-emptive case has to be utilised. As a rule of practical relevance, however, the highest importance value should be assigned to $v_{1}$, since the employees have to be enabled by their competence levels to select the appropriate activities to perform the tasks at the respective workplaces. The second step is to decide which workplace attributes should be covered. In the third step, it is necessary to assess the competence levels for each competence under consideration and each employee. Combining the competences and these competence levels yields the employee competence 
profiles. Moreover, the employees have to assess their preferences regarding the competences and the values of workplace attributes. The employees have to assess how much they prefer using one competence over another employing the AHP. In the fourth step, which can take place at the same time, the workplace requirement profiles have to be set up. Apart from the determination of the minimum required competence levels, the importance $w_{i j}$ of a competence $i$ to a workplace $j$ has to be assessed. The AHP is also employed for this assessment. Furthermore, the actual values of workplace attributes have to be assessed (e.g. the number of work days needed per week to complete the work specific to a workplace).

In the following table, all inputs needed to solve the goal programming model are listed:

Table 1. Input for the goal programming model

\begin{tabular}{|c|c|}
\hline$I$ & number of competences \\
\hline$J$ & number of workplaces \\
\hline$N$ & number of employees \\
\hline$K$ & number of workplace attributes \\
\hline$a_{i n}$ & actual level of competence $i$ for employee $n$ \\
\hline$b_{k j}$ & actual value of workplace attribute $k$ for workplace $j$ \\
\hline$g_{i j}$ & required level of competence $i$ for workplace $j$ \\
\hline$p_{\text {in }}$ & preference of employee $n$ regarding competence $i$ \\
\hline$h_{k n}$ & preferred value of workplace attribute $k$ for employee $n$ \\
\hline$w_{i j}$ & relative importance of competence $i$ to workplace $j$ \\
\hline$q_{k n}$ & importance of workplace attribute $k$ to employee $n$ \\
\hline$v_{1}$ & $\begin{array}{l}\text { importance of the fulfilment of the required competence } \\
\text { levels }\end{array}$ \\
\hline$v_{2}$ & $\begin{array}{l}\text { importance of the fulfilment of the employee's preferences } \\
\text { regarding the competences }\end{array}$ \\
\hline$v_{3}$ & $\begin{array}{l}\text { importance of the fulfilment of the employee's preferred } \\
\text { values of workplace attributes }\end{array}$ \\
\hline
\end{tabular}




\subsection{The Goal Programming Model}

\subsubsection{The Pre-emptive Case}

The first goal programming model presented below is utilised to establish the set of optimal assignments of employees to workplaces (shortly "optimal assignment sets" or "optimal solutions") which ensure that the respective workplace's competence level requirements are maximally fulfilled by the selected assignment sets. Each admissible assignment is represented by the decision variable $x_{j n}$ which is set to the value $1(0)$ if employee $n$ is (not) assigned to workplace $j$. If more than one assignment set maximally fulfils the competence level requirements, the preferences regarding both the competence levels and the values of (general) workplace attributes are additionally considered in a second goal programming model. The two types of employee preferences are weighted with their relative importance $v_{2}>0$ and $v_{3}>0$, respectively. The objective function (1) covers the objective of optimally assigning employees to workplaces according to their actual and required competence levels, respectively. This objective is viewed as the first-priority-goal, since the employees have to be able to select the appropriate activities to perform the tasks at the respective workplaces. The objective function only contains the underachievement variables $d_{i j}^{-}$as (individually weighted) first-priority-goal variables and not the overachievement variables $d_{i j}^{+}$of the required competence levels, since, in the example considered here, the objective is only to reach or to exceed the required competence levels (upper one-sided goals). Further, the objective function incorporates the AHP importance weights $w_{i j}$. Expressions such as those in equation (1) prevent a compensation of underachievements and overachievements of competence level requirements.

Objective function (first priority):

$$
\operatorname{MIN} \quad Z_{1}=\sum_{i=1}^{I} \sum_{j=1}^{J} w_{i j} * d_{i j}^{-}
$$

subject to the constraints:

$$
\sum_{n=1}^{N} a_{i n} * x_{j n}+d_{i j}^{-}-d_{i j}^{+}=g_{i j} \quad \text { for } \forall i=1, \ldots, I \quad \forall j=1, \ldots, J
$$




$$
\begin{aligned}
& d_{i j}^{+}, d_{i j}^{-} \geq 0 \quad \text { for } \forall i=1, \ldots, I \quad \forall j=1, \ldots, J \\
& N=J \vee N>J \rightarrow \sum_{n=1}^{N} x_{j n}=1 \quad \text { for } \forall j=1, \ldots, J \\
& N<J \rightarrow \quad \text { for } \forall j=1, \ldots, J \\
& \qquad \begin{array}{l}
\sum_{n=1}^{N} x_{j n} \leq 1 \\
N=J \vee N<J \rightarrow \sum_{j=1}^{J} x_{j n}=1 \quad \text { for } \forall n=1, \ldots, N
\end{array} \\
& \begin{array}{l}
\sum_{j=1}^{J} x_{j n} \leq 1 \quad \text { for } \forall n=1, \ldots, N \\
x_{j n}=\{0 ; 1\} \quad \text { for } \forall j=1, \ldots, J
\end{array} \quad \forall n=1, \ldots, N
\end{aligned}
$$

The underachievement variables $d_{i j}^{-}$and the overachievement variables $d_{i j}^{+}$guarantee that constraint (2) can always be fulfilled. From another point of view, constraint (2) ensures in connection with constraint (3) that the values of the deviational variables $d_{i j}^{-}$and $d_{i j}^{+}$are implicitly (model endogenously) determined. The "technical" non-negativity constraint (3) prevents the underachievement variables and the overachievement variables from becoming negative. Constraint (4) ensures that each workplace receives exactly one employee while constraint (5) ensures that each employee is assigned to exactly one workplace. The binary constraint (6) for all decision variables $x_{j n}$ completes the set of constraints in this model. If the (first) goal programming model above leads to more than one optimal solution, the (second) model below considering additionally the second-priority-goals has to be solved. The expression in the first row of the objective function (7) considers the deviations of the relative importance of the competences (for each assigned workplace $j$ ) from the employee preferences regarding the competences $p_{i n}$. This expression contains the underachievement variables $d_{i n}^{-}$as well as the overachievement variables $d_{i n}^{+}$, since the objective is to meet exactly the preferences (two-sided goals). In the second row of the 
objective function (7), the deviations of actual values $b_{k j}$ (for each assigned workplace $j$ ) from preferred values $h_{k n}$ of each workplace attribute $k$ are considered for each employee $n$. Since the preferred values should be met exactly (two-sided goals), such as hours of work per day, and number of work days in a week, underachievement variables $d_{k n}^{-}$and overachievement variables $d_{k n}^{+}$are used once again. Other workplace attributes may be considered as one-sided goals. Some workplace attributes may require that a certain level is reached or exceeded. Examples of these workplace attributes are operational safety and office space. Other workplace attributes may need to be achieved equal to or below a certain level. Examples of such workplace attributes are air and noise pollution. To simplify matters, the objective function (7) does not take into account attributes viewed as one-sided goals, but it can easily be adjusted to do so.

The constraints (4) to (6) of the first model dedicated only to first-priority-goals also apply for the second model considering additionally the second-priority-goals. The constraints (8) and (9) ensure in connection with constraints (10) and (11), respectively, that the values of the deviational variables $d_{i n}^{+}, d_{i n}^{-}, d_{k n}^{+}$, and $d_{k n}^{-}$are implicitly (model endogenously) determined. The non-negativity constraints (10), and (11) prevent the deviational variables $d_{i n}^{+}, d_{i n}^{-}, d_{k n}^{+}$, and $d_{k n}^{-}$from becoming negative. Constraint (12) ensures that the maximal level $Z_{1}^{*}$ of weighted fulfilment of all first-priority-goals which is realised by all optimal solutions of the first model also holds in the second model. Therefore constraint (12) plays the role of an integrity condition for consistently linking the second to the first model of the same real assignment problem.

Objective function (second priority):

$$
M I N\left[\begin{array}{l}
v_{2} *\left(\sum_{i=1}^{I} \sum_{n=1}^{N} d_{i n}^{-}+d_{i n}^{+}\right) \\
+v_{3} *\left(\sum_{k=1}^{K} \sum_{n=1}^{N} q_{k n} *\left(d_{k n}^{-}+d_{k n}^{+}\right)\right.
\end{array}\right]
$$

subject to the constraints:

$$
\sum_{j=1}^{J} w_{i j} * x_{j n}+d_{i n}^{-}-d_{i n}^{+}=p_{i n} \quad \text { for } \forall i=1, \ldots, I \quad \forall n=1, \ldots, N
$$




$$
\begin{aligned}
& \sum_{j=1}^{J} b_{k j} * x_{j n}+d_{k n}^{-}-d_{k n}^{+}=h_{k n} \quad \text { for } \forall k=1, \ldots, K \quad \forall n=1, \ldots, N \\
& d_{i n}^{+}, d_{i n}^{-} \geq 0 \quad \text { for } \forall i=1, \ldots, I \quad \forall n=n, \ldots, N \\
& d_{k n}^{+}, d_{k n}^{-} \geq 0 \quad \text { for } \forall k=1, \ldots, K \quad \forall n=1, \ldots, N \\
& Z_{1}^{*}=\sum_{i=1}^{I} \sum_{j=1}^{J} w_{i j} * d_{i j}^{-}
\end{aligned}
$$

\subsubsection{The Non-pre-emptive Case}

The non-pre-emptive goal programming model presented below is capable of considering all three objectives simultaneously. By contrast to the preemptive case, the following non-pre-emptive case allows compensation effects between the three objectives.

The objective function (11) seeks the optimal solutions of the assignment model each of which maximally fulfills the sum of weighted fits between two complementary aspects. Each row of the objective function (11) represents one of the three objectives. As in the pre-emptive case, the first objective refers to upper-one-sided goals, while the second and the third objectives refer to two-sided goals. The first row covers the assignment of the employees to workplaces according to the weighted $\left(v_{1}\right)$ fit between weighted $\left(w_{i j}\right)$ actual and required levels of competence for employees versus workplaces, respectively. The expression in the second row of the objective function (11) considers the weighted $\left(v_{2}\right)$ fit of the employee preferences regarding the competences and the relative importance of the competences to the workplaces, while the expression in the third row covers the weighted $\left(v_{3}\right)$ fit between weighted $\left(q_{k n}\right)$ preferred versus actual values of workplace attributes. It is possible that there are several different assignment sets meeting the competence level requirements and preferences in the same optimal way, i.e., with the same maximal value of the objective function (11). Especially compensation effects between the three objectives can lead to several optimal solutions of the assignment model. 


$$
M I N\left[\begin{array}{l}
v_{1} *\left(\sum_{i=1}^{I} \sum_{j=1}^{J} w_{i j} * d_{i j}^{-}\right) \\
+v_{2} *\left(\sum_{i=1}^{I} \sum_{n=1}^{N} d_{i n}^{-}+d_{i n}^{+}\right) \\
+v_{3} *\left(\sum_{k=1}^{K} \sum_{n=1}^{N} q_{k n} *\left(d_{k n}^{-}+d_{k n}^{+}\right)\right.
\end{array}\right]
$$

The constraints (2) to (6) and (8) to (11) from the pre-emptive case also apply in the non-pre-emptive case.

\section{DIFFICULTIES OF THE MODEL}

One obvious problem could be that the input data is not updated regularly. Hence, for example, the documentation of the competence profiles and the preferences of the employees might be out of date or incomplete. This may cause misleading assignment sets. Another problem may occur if the employee to be assigned has irreconcilable differences with another employee assigned to a workplace in the vicinity of the first employee's workplace, so that the enforced cooperation demotivates both employees and thus outweighs the efficiency gains of the workplace assignment delivered by the goal programming model. Moreover, the employee to be assigned may be content with her or his present workplace and may thus dislike an assignment to another workplace. This might be the case when the competence levels required for her or his present workplace deviate greatly from her or his actual competence levels or when his or her preferred competence levels are neglected or assessed to have low relative importance. One way to deal with this problem is to ask the employees, before utilising the goal programming model, whether they agree with an assignment to another workplace. In that case, only the employees agreeing with the assignment to another workplace may be considered in the goal programming model. If the employee's actual competence levels do not fulfil his present workplace's competence level requirements, but his or her preferred competence levels match the required competence levels of the workplace, another solution to this problem may be to offer the employee training opportunities with respect to the required competence levels. 


\section{CONCLUDING REMARKS}

This paper has presented a formal model for the real problem of assignment of employees to workplaces considering workplace competence level requirements, importance of competences to workplaces, and actual workplace attribute values on the one hand and the employee's actual and preferred competences and preferred workplace attribute values on the other hand. The optimal solution of this model delivers at least one assignment set minimising the sum of the weighted discrepancies firstly caused by the weighted deviations between competence levels required for a workplace and the actual competence levels of the employees, secondly caused by the deviations between the importance of competences to a workplace and the employees' preferences regarding the competences, as well as thirdly caused by the weighted deviations between the actual workplace attributes and the employees' preferred values of the workplace attributes. The minimisation of the sum of the weighted discrepancies mentioned before equals to the maximal weighted fulfilment of workplaces' competence level and (general) attribute value requirements by each optimal assignment of employees to workplaces.

\section{ACKNOWLEDGEMENTS}

This work was partly funded by the Federal Ministry of Education and Research (BMBF) and coordinated both by the German Aerospace Centre (DLR) within the project "MOTIWIDI" and the Research Centre Karlsruhe (PFT) within the project "KOWIEN".

\section{REFERENCES}

CHARNES, Abraham; COOPER, William W.:

Management Models and Industrial Applications of Linear Programming, Volume I.

New York, London, Sydney: John Wiley \& Sons, 1961.

HILLIER, F. S.; LIEBERMAN, G. J.:

Introduction to Operations Research.

Boston, MA: McGraw-Hill, 7th ed., 2001.

HOFFMAN, J. J.; SCHNIEDERJANS, M. J.:

Two-stage Model for Structuring Global Facility Site Selection Decisions.

In: International Journal of Operations \& Production Management,

Bradford, 14(1994)1, pp. 79-96. 
MUKHERJEE, K.; BERA, A.:

Application of goal programming in project selection decision - A case study from the Indian Coal mining industry.

In: European Journal of Operational Research,

Amsterdam, 82(1995)1, pp. 18-25.

SAATY, Thomas L::

Decision Making for Leaders.

Pittsburgh, PA: RWS Publications, 3rd Edition, 2001. 